\title{
The program Formal_Charges
}

\author{
Version July 2018, Nebil A. Katcho - ILL
}

The program Formal_Charges is a console utility for converting CIF files to CFL files that allows Bond-Valence Energy Landscapes calculations with the program BondStr. The program is fully based in CrysFML (Crystallographic Fortran 95 Modules Library).

The program needs an input file that can be either a standard CIF file or a CFL file containing just the necessary structural information. The input file can be given as a command-line argument that is the name of the file to be processed. If the file has the extension BUF, the program assumes that the input file contains a list of CFL or CIF files to be processed. In this last case another buffer file is created to be treated by BondStr program.

If the program is invoked without command-line argument it asks for the name of a CIF or CFL file to be treated. Example:

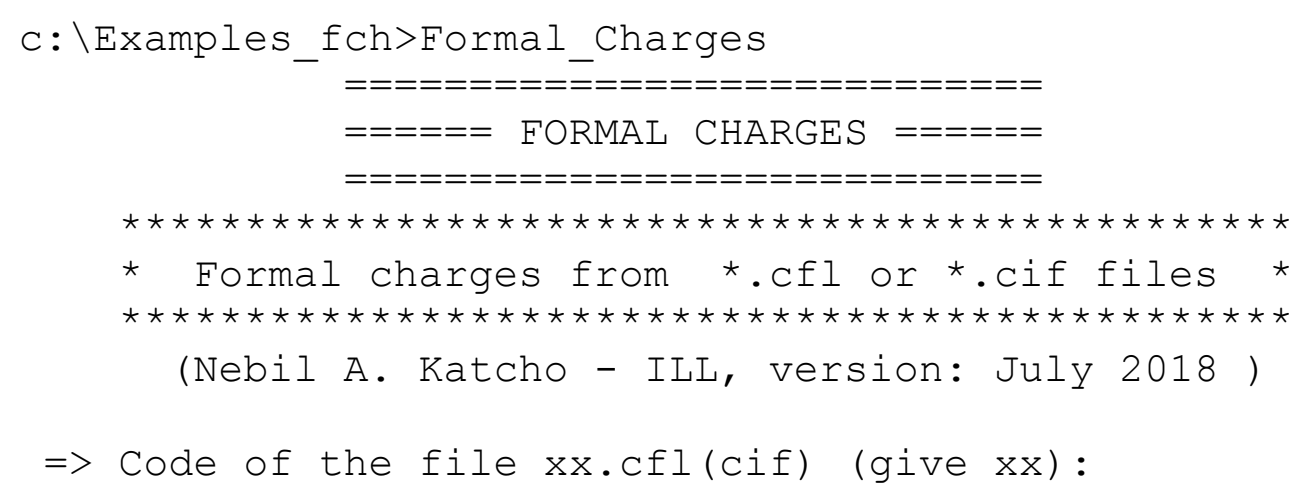

The use should give a valid code $(\mathrm{xx})$ existing in the current directory. If not the program stops after giving a message:

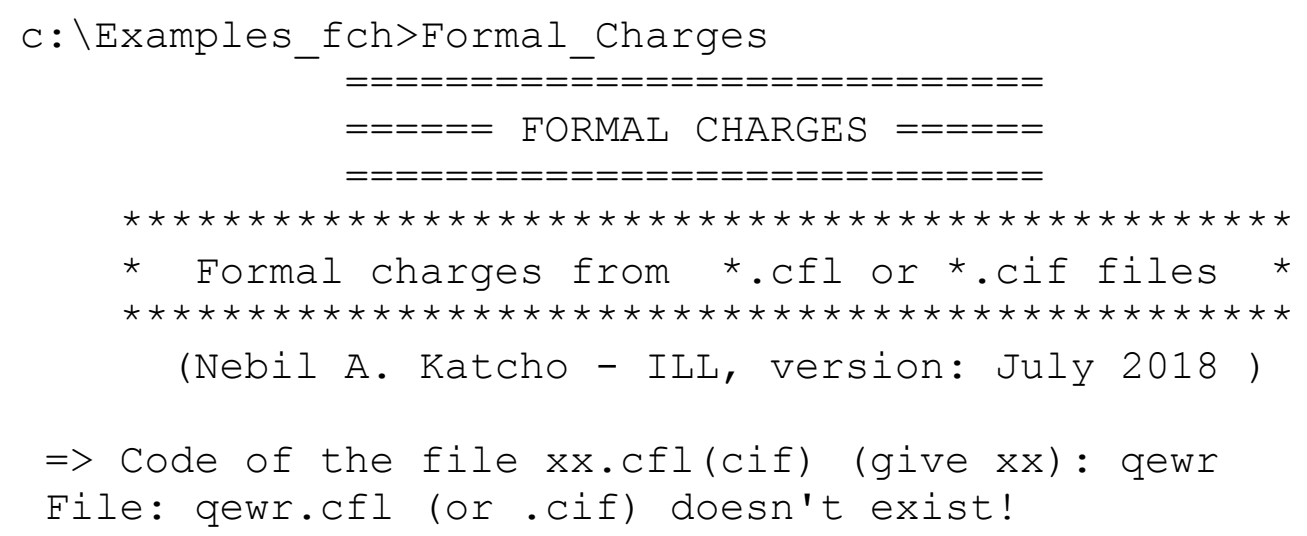

The normal way of invoking the program is providing a command-line argument: c: $\backslash$ Examples_fch>Formal_Charges NiFePO5_gen.cif 


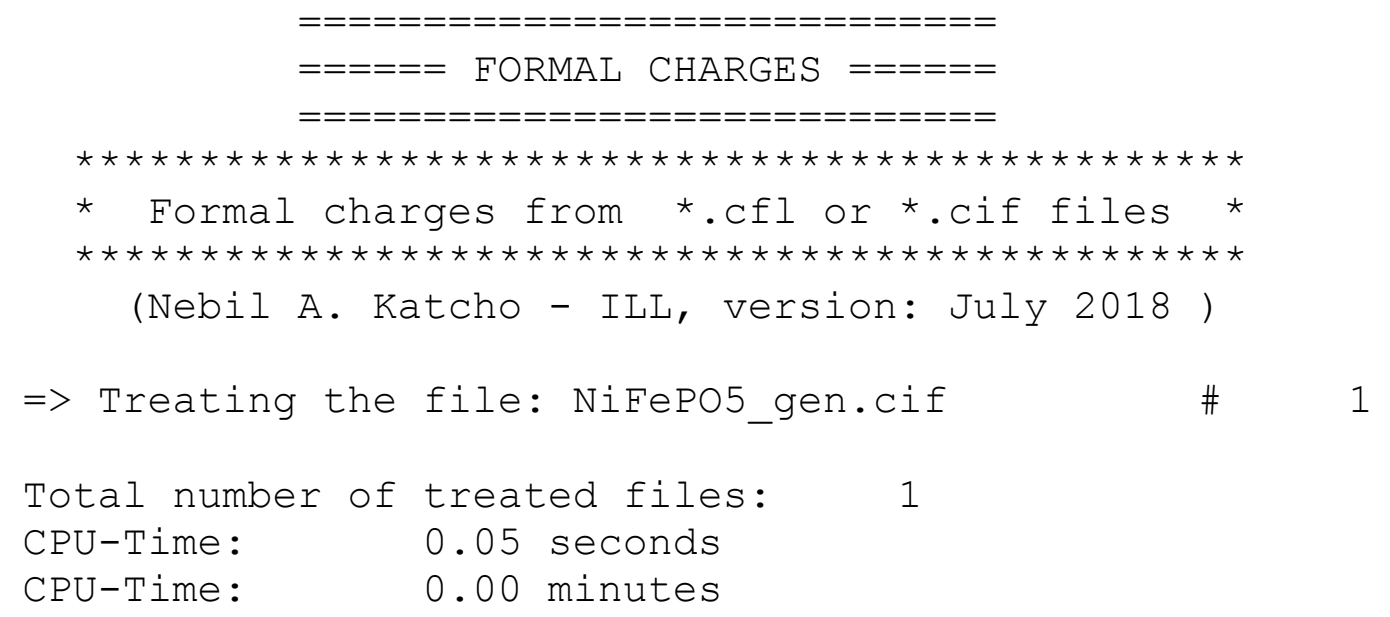

After running the program a file with extension $\mathrm{FCH}$ (in this case NiFePO5_gen. $\mathrm{fCh}$ ) is generated containing the results of the treatment. The content of this file is self-explanatory.

The most important use of this utility is for treating many CIF files for generating a buffer file with CFL files containing all the proper information to run Bond-Valence Energy Landscapes calculations using BondStr. An example of this use is given below.

C: \Database \CIFs>Formal_Charges buffer_small.buf

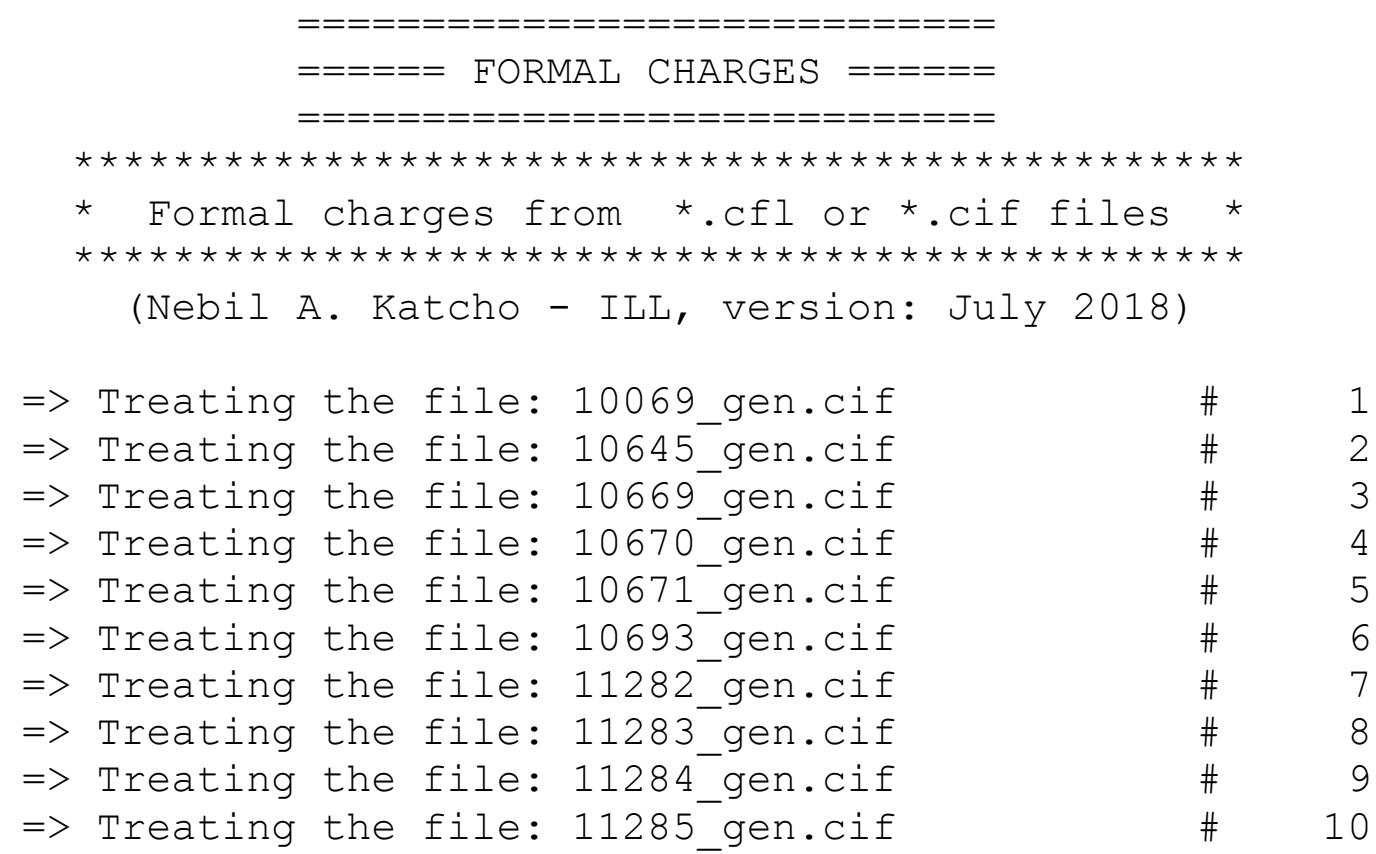

Total number of treated files: 10

CPU-Time: $\quad 0.23$ seconds

CPU-Time: $\quad 0.00$ minutes

After running Formal_Charges using a buffer file containing the names of CIF files the program creates CFL files and a buffer file ( $\mathrm{cfl}$ _buffer.buf) containing the generated CFL filenames. In our case the content of $\mathrm{cfl}$ _buffer.buf is: 


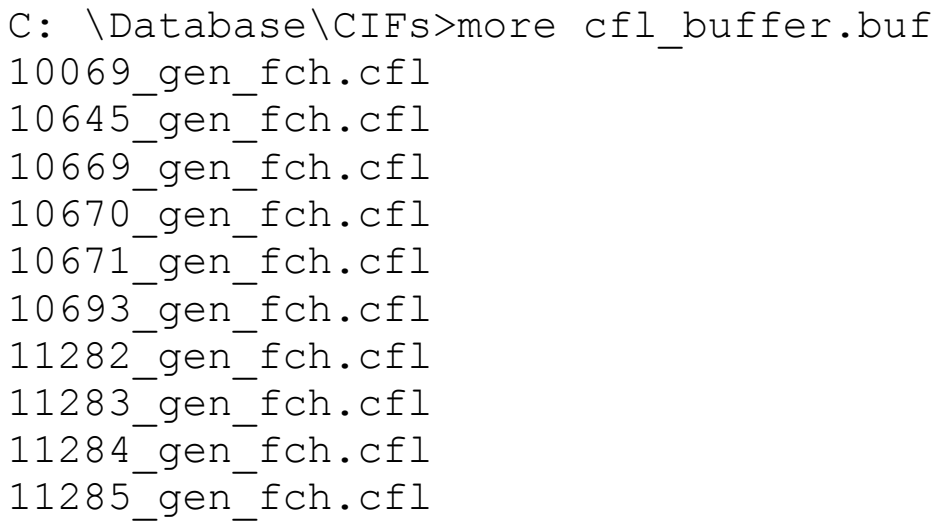

Notice that the filenames contain the suffix _ $f \mathrm{Ch}$ for indicating the origin of the CFL files. The content of the generated CFL file depends on additional arguments provided in the command line. In our case only the name of the buffer file has been provided so the content of the CFL file by default is similar to the following:

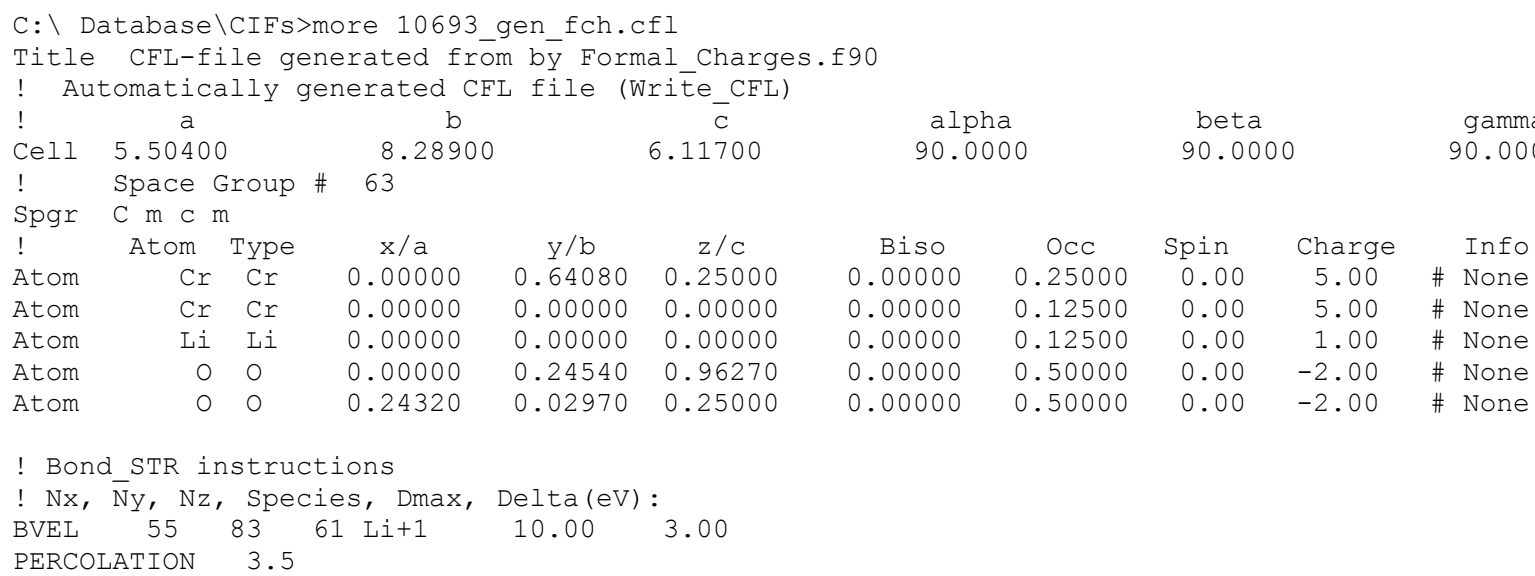

By default the program determines the chemical species that is probably the mobile ionic species in the given chemical compound. The user can change that by adding a second argument in the command line corresponding to the desired ionic species. For instance invoking the program as:

C: \Database \CIFs>Formal_Charges buffer_small.buf $\mathrm{Na}+1$

Moreover if the user wants to use soft bond-valence parameters in BondStr an argument containing the keyword SOFTBVS should be given as in:

C: \Database\CIFs>Formal_Charges buffer_small.buf SOFTBVS

If both the chemical species and soft BVS parameters are to be used, the chemical species name should be given before the keyword SOFTBVS as in:

C: \Database \CIFs>Formal_Charges buffer_small.buf Na+1 SOFTBVS

The CFL files can be used directly to perform BVEL calculations by BondStr that admits also the buffer file containing CFL files generated by Formal_Charges. 